\title{
Description of a Framework to Enhance Antiretroviral Therapy Adherence using Self-efficacy and Belief about Medicines
}

\author{
Adegoke O. Adefolalu
}

\begin{abstract}
There is ample evidence that strict adherence to antiretroviral therapy (ART) is strongly associated with optimal health outcomes among people living with HIV (PLWHA), although strict adherence to treatment regime is exceedingly difficult in chronic medical conditions. The intention to adhere to ART among PLWHA is often influenced by individual adherence self-efficacy, along with their Beliefs about Medicines. Various studies have described the factors influencing adherence in ART, and most of these were guided by conceptual framework encompassing the relevant constructs of the Cognitive-Behavioural theory (CBT), in which health behaviour is viewed as a result of an individual's conscious rational choice. Based on the results of an empirical study conducted among a cohort of PLWHA and the findings from the literature, this paper describes a framework for enhancing adherence to ART using the Self-Efficacy model and Belief about Medicines theory. These two constructs from the CBT have been widely used in studying patients' adherence to longterm therapy in chronic medical conditions. The identified interconnected system of beliefs associated with ART adherence are used in developing an algorithm which will prompt a healthcare professional to initiate need-based interventions that seeks to reduce personal beliefs that are negatively influencing the person's adherence to ART.
\end{abstract}

Index Terms - antiretroviral therapy; adherence framework; Self-efficacy; Belief about medicines.

\section{INTRODUCTION}

Self-Efficacy is one of the constructs described under the Cognitive-Behavioural Theories (CBT) that are used by the health behaviorists to understanding adherence to chronic medical conditions [1], [2]. In the context of change in behaviour of a person concerning their health and disease, Self-Efficacy describes the person's belief that they can alter certain behaviour or carry out a specification required, to achieve certain positive health outcomes in the process of managing a disease condition [1], [3]. In addition, SelfEfficacy has been shown to indicate how patients with chronic medical conditions will behave in terms of adhering to medications including ART adherence [4] - [9]. Therefore, Adherence Self-Efficacy can be defined as the individual's belief in the ability to strictly adhere to ART despite any challenges to the person's health or circumstance [1]. This concept is increasingly becoming important in assisting patients with chronic disease, it entails how one can acquire the necessary skill that will enable the individual to develop good self-care behaviour. Self-Efficacy continues to be relevant in the context of being recognized as an important construct of Social Cognitive Theory (SCT), which postulates that people with high Self-Efficacy that they can carry out certain health behaviour are more likely to perform such behaviour [1], [2], [10].

Belief about Medicines is another concept in Cognitive Behavioural Theories that is also used in studying adherence to chronic medical conditions [1], [2]. According to the literature, a cost-benefit analysis is usually undertaken by individuals on long term treatment, whether their beliefs about the necessity of using medicines to maintain their health in good status outweigh their concerns about the potential side effects of using the medicines [11], [12]. Therefore, the resultant action taken by such person after doing the cost-benefit analysis is what is described as one's' beliefs about the medicine. The Beliefs about Medicines Questionnaire (BMQ) was developed in response to the above assertion, it was postulated that a specific measure to gauge patients' beliefs about medicines would lead to an enhanced understanding of patients' beliefs about their medications [13]. In addition, it was also assumed that improved understanding of patient's beliefs about their medications could inform the development of certain interventions that could be used to enhance adherence, and further optimize the benefits that patients get from using medicines [13]. The Human Immunodeficiency virus (HIV) and Acquired Immune deficiency Syndrome (AIDS) continue to be one of the challenging public health concern of this generation, affecting all age groups, causing premature deaths, and depleting both human and capital resources in the worst affected areas. Globally, about 40 million people are living with HIV, although no definite cure has been found to treat this infectious disease, the introduction of antiretroviral therapy has enabled the disease to be managed as a chronic life-threatening disease. Various studies in resourceconstraint settings have shown that high levels of adherence, viral suppression and good clinical outcome are achievable in these settings but strict adherence to treatment regime is exceedingly difficult in chronic medical conditions. Research has shown that patients' adherence self-efficacy and medication beliefs can be altered through focused intervention [14]. HIV-infected patients on ART will choose to adhere to their medication if they believe that doing so will result in getting better and living quality life [1]. HIV-

Published on July 11, 2020.

A.O. Adefolalu, Sefako Makgatho Health Sciences University, South Africa

(corresponding e-mail: adegoke.adefolalu@ ${ }^{@}$ smu.ac.za). 
infected patients need to have confidence in their ability to perform the required self-management activities (selfefficacy) and hold positive beliefs about their medications [1], [8], [9]. This framework is based on the premise that exhibiting self-care behaviour and holding the right beliefs about medications lead to good clinical outcomes which translate to quality health.

Developing a framework that will enhance adherence to antiretroviral therapy is necessary to assist patients' in attaining competence in Self-Efficacy and to develop positive beliefs about medications, which would eventually lead to improved adherence to therapy and a subsequent better clinical outcomes on lifelong ART. There is enough empirical evidence in the literature and in the previous study that selfefficacy and perceived general harm and overuse of medicines, are among the cognitive factors that influence adherence to ART [1], [4] - [9]. The framework described here is based on the findings of a previous research and focuses on the individual behaviour assuming that it is shaped by cognitions such as attitudes, beliefs, motivations and selfefficacy [15], [16] The researcher proposed that attention should be given to these cognitive-behavioural variables that influence adherence to treatment.

\section{METHODS}

\section{A. Study design and procedure}

The study employed a quantitative research design using some quantitative instruments to measure the cognitive variables among some HIV-infected persons on ART. Ethical clearance was obtained from the Higher Degrees Committee of the Department of Health Studies, University of South Africa (HSHDC 62/2011). As mentioned earlier, the results of the research have been published earlier [15], [16] The research examined the relationships between two individual factors (self-efficacy and beliefs about medicines) and the health behaviour (HIV medication adherence) in an attempt to identify the potential area for focused interventions that could be used in developing a framework for enhancing ART adherence [15]. This research was based on the assumptions that possessing a high level of self-efficacy towards ART adherence and positive beliefs about medicines will increase the likelihood that an individual would be more motivated to actively achieve high adherence to ART and good clinical outcome [15].

\section{B. Framework for Enhancing ART Adherence}

Fig. 1 represents the proposed modality of enhancing adherence to ART focusing on self-efficacy and Beliefs About Medicines. It shows the goals, intervention and outcome of the intervention that aim to improve patients' adherence Self-Efficacy and stronger beliefs about Medicine (i.e. general harm and overuse of medicines). The framework for enhancing ART adherence among HIV-infected patients comprises of the following structure: Purpose of the framework, Assumptions of the framework and the Participants within the framework.

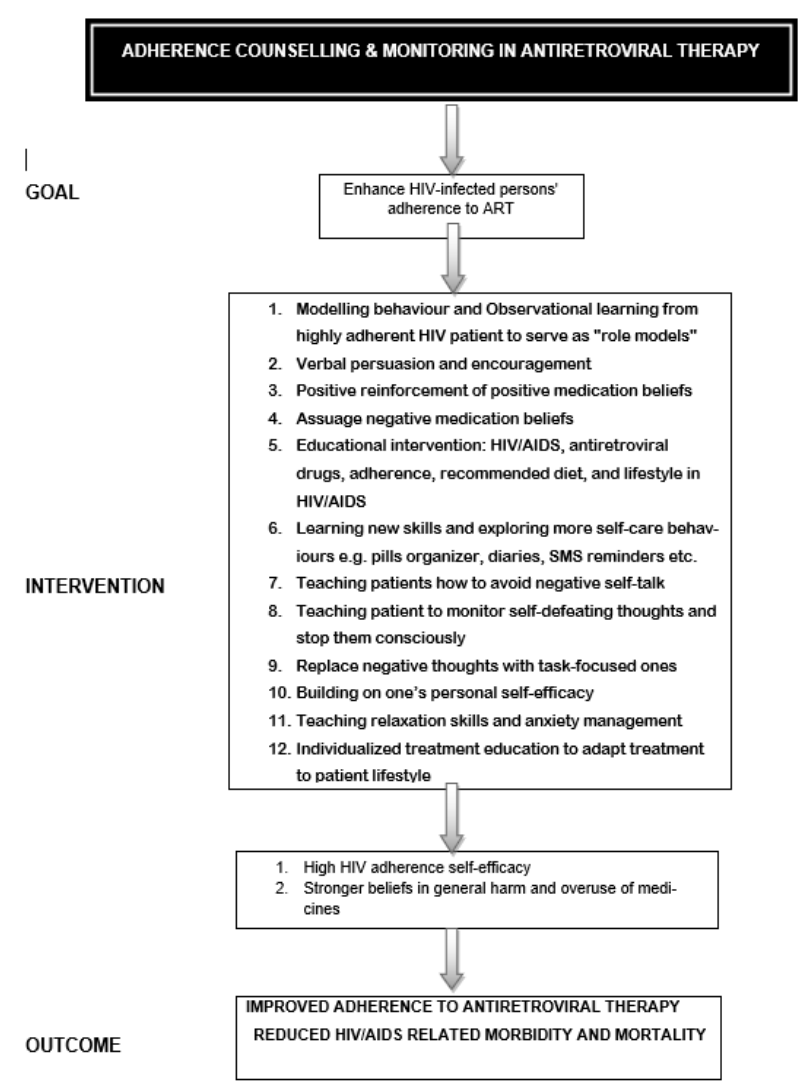

Fig. 1. A Model of the visual flowchart diagram of ART adherence enhancement.

\section{Purpose of the framework}

The purpose of the framework is to provide modalities for adherence counsellors and other health care providers involved in HIV management on how to enhance adherence of patients on antiretroviral therapy using the identified cognitive factors in this study. The major aim of the framework is to improve adherence to ART among patients thereby reducing the morbidity and mortality associated with HIV/AIDS. The research findings revealed that HIV adherence self-efficacy is a predictor of adherence to ART among patients who are non-adherent to antiretroviral therapy and general beliefs about medicines is a predictor of ART adherence among HIV-infected patients on ART. In addition, there is inter-relationship between adherence self-efficacy and general beliefs about medicines; and between specific beliefs about medicines and general beliefs about medicines [15].

\section{Assumptions of the framework}

HIV/AIDS like any other chronic medical conditions requires that patients should be made to go through some counselling sessions before being initiated on antiretroviral treatment. Since ART is a lifetime commitment, it is particularly important to counsel a patient adequately before initiation of treatment. The assumptions of this framework are based on the following in cognizance of ART:

1. Adequate counselling will precede initiation of ART initiation.

2. The process of adherence counselling will identify the potential barriers and facilitators of adherence before initiation of ART. 
3. Adherence monitoring and counselling is a continuum and will not be treated as once-off event in antiretroviral therapy.

\section{E. Participants within the framework}

This is a framework for enhancing ART adherence among patients. However, other stakeholders will be involved to achieve the desired outcome of this framework. Participants in the framework will include HIV-infected patients on ART, Health care providers, and Treatment buddies and a family member of patients. It is important to include people with whom patients on ART would collaborate in the framework. All these stakeholders work in an interactive fashion to enhance adherence by assisting the patient to change the unrealistic expectations or behaviour through education that helps to learn how to do things in a new or different ways. Patients can control or influence the events that affect their lives by integrating cognitive, social, and behavioural subskills related to beliefs of personal efficacy in performing these skills.

\section{F. Framework's overview and structural description}

The proposed framework encompasses HIV-ASES, BMQG, BMQ-S and ACTG questionnaires to be used in adherence counselling to enhance adherence among HIV-infected patients on ART. Fig. 2 is a structural representation of the framework. The oval structures (Marked black) on the left side represent the potential facilitators and barriers to ART adherence namely:

- Socio-demographic characteristics, accessibility to health services, social support;

- HIV adherence self-efficacy (HIV-ASES);

- Perceived general harmful effects and overuse of medications (BMQ-G);

- $\quad$ Specific necessity and concerns about ARVs (BMQ-S).

The solid pillar-shaped column on the far-right side represents adherence to ART which is the desired outcome of antiretroviral therapy use. The arrows pointing to the right indicate direction of the activities that lead to adherence. The up-down arrows (marked brown) indicate interrelationship between certain cognitive variable and the down-arrow callout (marked blue) depicts the interventions carried out after potential barriers to adherence have been identified. The enhancement of adherence is achieved through the intervention targeted at the identified barrier or identified facilitator of adherence.

\section{STEP 1: BASELINE SCREENING FOR ADHERENCE FACILITATORS AND BARRIERS}

The kick-starting of the series of activities in the framework begins at this point when patients are being counselled and empowered with the necessary information about the antiretroviral therapy processes. The oval structures in Fig. 2 represent the factors influencing ART adherence. The goal at this stage is to identify the cognitive-behavioural factors that will influence an individual in adhering to ART. Fig. 3 below shows the graphical representation of the start of the first phase of the entire process where baseline screening for factors influencing ART adherence is conducted followed by scoring of patients on the two scales. At this stage two instruments are needed to identify these factors, the 1st one is the HIV-ASES (this assesses the adherence self-efficacy) and the second one is the BMQ-G (this assesses the general harm and overuse of medicines).

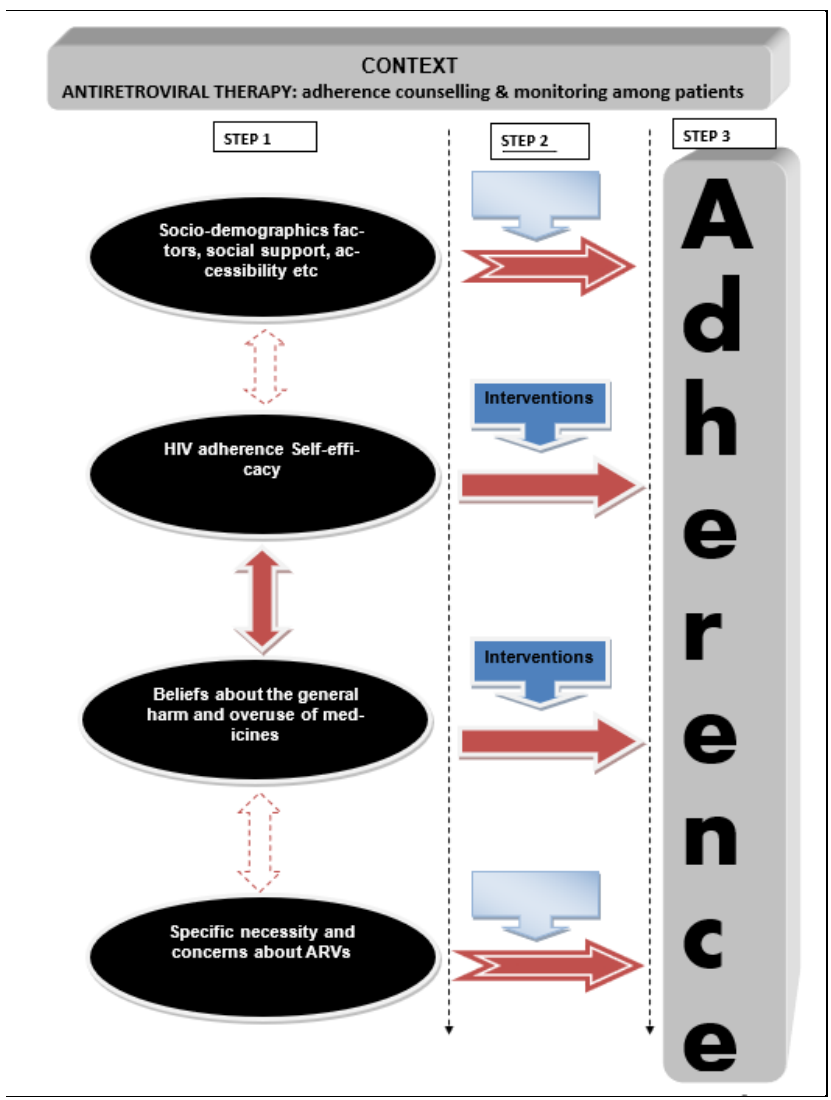

Fig. 2. A Visual graphical representation of the ART adherence framework

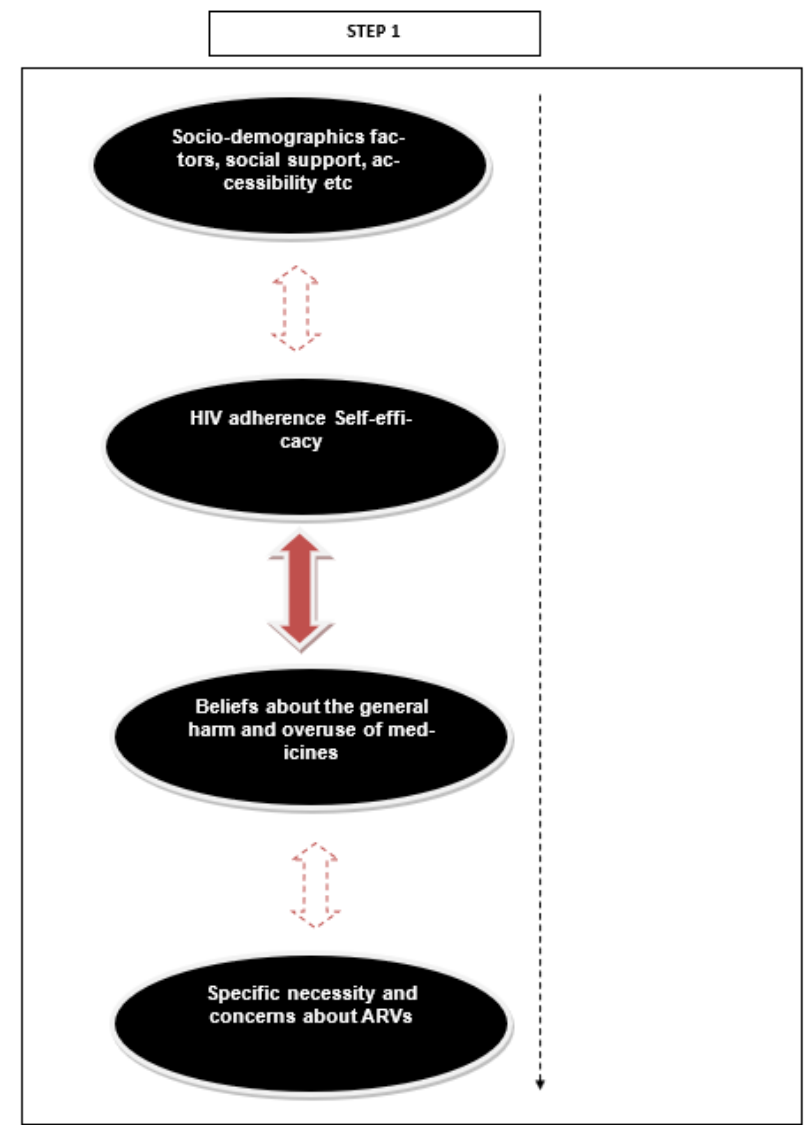

Fig. 3. Baseline screening for factors influencing adherence and scoring of patients. 
The HIV-ASES (Table I) is used to assess the confidence of the patients in their ability to carry out the tasks outlined in Table 1 below. The performance of these tasks results in the desired outcome which is adherence to ART. The patients are asked the 12 questions on the HIV-ASES and each item is scored between $0-10$, the average score is then calculated (Total score $\div 12$ ), the optimal score is 10 . Any patient that scores below 5 points on this scale is identified as a potential non-adherent person, and such individual is prioritized for intervention.

TABLE I: HIV ADHERENCE SELF-EFFICACY SCALE (HIV-ASES) [8]

\begin{tabular}{|c|c|c|}
\hline & HIV Adherence Self-Efficacy Scale (HIV-ASES & $\begin{array}{c}\text { Score } \\
0-10\end{array}$ \\
\hline 1 & $\begin{array}{l}\text { Stick to your treatment plan even when side effects begin } \\
\text { to interfere with your daily activities? }\end{array}$ & \\
\hline 2 & Integrate your treatment into your daily routine? & \\
\hline 3 & $\begin{array}{l}\text { Integrate your treatment into daily routine even if it means } \\
\text { taking medication or doing other things in front of people } \\
\text { who don't know you are HIV+ }\end{array}$ & \\
\hline 4 & $\begin{array}{l}\text { Stick to your treatment schedule even when your daily } \\
\text { routine is disrupted? }\end{array}$ & \\
\hline 5 & $\begin{array}{l}\text { Stick to your treatment schedule when you aren't feeling } \\
\text { well? }\end{array}$ & \\
\hline 6 & $\begin{array}{l}\text { Stick to your treatment schedule when it means changing } \\
\text { your eating habits? }\end{array}$ & \\
\hline 7 & $\begin{array}{l}\text { Continue with your treatment even if doing so interferes } \\
\text { with your daily activities? }\end{array}$ & \\
\hline 8 & $\begin{array}{l}\text { Continue with the treatment plan your physician } \\
\text { prescribed even if your CD4 count drop significantly in } \\
\text { the next three months? }\end{array}$ & \\
\hline 9 & $\begin{array}{l}\text { Continue with the treatment plan your physician } \\
\text { prescribed even if your CD4 count drop significantly in } \\
\text { the next three months? }\end{array}$ & \\
\hline 10 & $\begin{array}{l}\text { Continue with your treatment even when getting to your } \\
\text { clinic appointments is difficult? }\end{array}$ & \\
\hline 11 & $\begin{array}{l}\text { Continue with your treatment even when people close to } \\
\text { you tell you that they don't think that it is doing any good? }\end{array}$ & \\
\hline 12 & $\begin{array}{l}\text { Get something positive out of your participation in } \\
\text { treatment, even if the medication you are taking does not } \\
\text { improve your health? }\end{array}$ & \\
\hline \multicolumn{2}{|r|}{ Total } & \\
\hline
\end{tabular}

The BMQ-G (Table II) is used to screen for beliefs about the general harm and overuse of medicines. The patient's average score is recorded based on the 5-point Likert scale with responses ranging from strongly agree -1 , agree -2 , uncertain -3 , disagree -4 , to strongly disagree -5 , and the optimal score is 8 points. Any patient that scores below 4 points on this scale is identified as a potential non-adherent person, and such person is prioritized for further intervention.

TABLE II: Beliefs About Medicines QuestionnAire-GenERAL (BMQ-G)

\begin{tabular}{|l|c|c|c|c|c|}
\hline \multicolumn{1}{l}{$[13]$} & S/A & A & U & D & S/D \\
\hline Doctors use too many medicines & 1 & 2 & 3 & 4 & 5 \\
\hline $\begin{array}{l}\text { People who take medications should stop } \\
\text { their treatment for a while every now and } \\
\text { again }\end{array}$ & 1 & 2 & 3 & 4 & 5 \\
\hline Most medicines are addictive & 1 & 2 & 3 & 4 & 5 \\
\hline Natural remedies are safer than medicines & 1 & 2 & 3 & 4 & 5 \\
\hline Medicines do more harm than good & 1 & 2 & 3 & 4 & 5 \\
\hline All medicines are poisons & 1 & 2 & 3 & 4 & 5 \\
\hline $\begin{array}{l}\text { Doctors place too much trust on } \\
\text { medicines. }\end{array}$ & 1 & 2 & 3 & 4 & 5 \\
\hline $\begin{array}{l}\text { Doctors place too much trust on } \\
\text { medicines. }\end{array}$ & 1 & 2 & 3 & 4 & 5 \\
\hline \multicolumn{1}{|c|}{ Total } & & & & & \\
\hline
\end{tabular}

S/A - Strongly agree. A - Agree. U - Uncertain. D - Disagree. S/D - Strongly disagree

\section{STEP 2: FOCUSED INTERVENTIONS ON IDENTIFIED BARRIERS OF ART ADHERENCE}

The main objective at this stage is to address the identified factors in step 1 that could serve as barriers to ART adherence. The framework proposes minimum score on the BMQ-G as 4, and minimum score on the HIV-ASES as 5. Patients that score below these cut-off marks are categorized as possessing cognitive-behavioural factors that serve as barriers to ART adherence. This is then followed by defining the identified factors and communicating them to the patient for intervention. Subsequent to this is setting of goals and strategies for the intervention according to the identified psychological and physical needs of the patient. Fig. 4 shows a schematic diagram of the identified cognitive variables. The arrows pointing to the right indicate the desired outcome for the patient and the down-arrow callout (marked blue) marks the point of intervention outlined for a specific patient. The activities that could be used at this stage include but not limited to the following:

1. Address the identified cognitive variables in the scales where the individual scores low points by education, counselling, skills development and provide practical assistance where necessary. This may involve discussion with other members of the health team on the need to review ART regimen, schedule etc.

2. Review the individual perception of health goal, HIV disease, purpose of antiretroviral therapy, benefits of adherence and consequences of non-adherence to ART.

3. Educate the individual further on the facts about ART adherence, factors influencing adherence and consequences of non-adherence.

4. Assist the patient to assume a participatory role in decision made about his/her health especially in the timing of medications intake, regimens that suit his/her daily routine etc.

5. Assist the individual to develop a greater sense of selfcare behaviour, setting personal goals and implementing strategies to achieve the set goals.

6. Developing skills to incorporate treatment needs into their lifestyle.

7. Assist the patient in terms of self-management training for taking daily medications, motivational interview, and social support, if necessary.

8. Reminder devices such as pillboxes, pill chart, medication diaries, beepers, and alarms etc. may be provided for certain individuals, depending on their circumstances.

Learning occurs through information acquisition and is often interactive. This stage emphasizes the need for learning through feedback and internalization of the processes that lead to behavioural change. Patients need to demonstrate self-reinforcement and self-enhancement through feedback to the adherence counselling and ability to develop an action plan to achieve improved adherence to ART. The development of cognitive-behavioural competency skills, such as adherence self-efficacy and beliefs about medicines in HIV management at this stage will result in better adherence. 


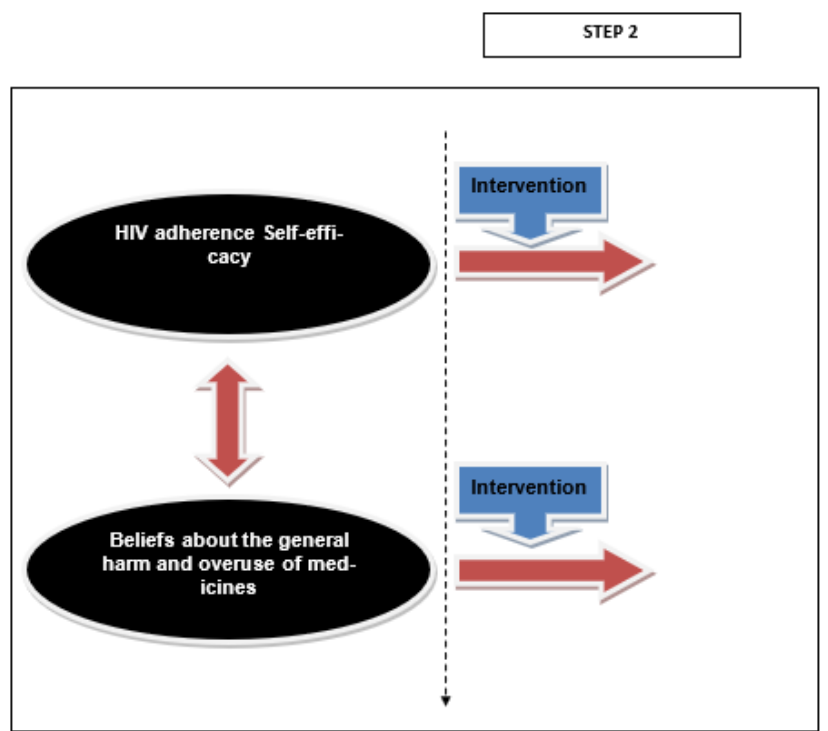

Fig. 4. Focused interventions on identified barriers to ART adherence is done here.

\section{STEP 3: OUTCOME OF FOCUSED INTERVENTION- ADHERENCE TO ART}

Fig. 5 depicts the diagrammatic picture of what occurs in step 3. The solid column on the far right represents adherence to ART that is achieved by cognitive-behavioural interventions carried out in earlier stage. The ACTG questionnaire is used at this stage and it determines the medication adherence to prescribed ARVs in the previous four days, together with the compliance of the patient with dosing schedule and the extent to which the patient has followed dietary instructions for ARV use in the previous four days.

The aim at this stage is to emphasize the importance of adherence to ART following development of competency in self-efficacy and possession of stronger beliefs in the use of medicines. The achievement of this competency will result in sustained adherence to antiretroviral therapy which will be evident in reduced HIV/AIDS morbidity and mortality. Competent individuals are more self-directed and goal oriented which lead to self-actualization. The competent patient has the ability to cope with encountered situations and maintain self-care management skills at all time. 3

The framework described above can be summarized as follows:

- Step 1: an HIV-infected patient is screened for cognitivebehavioural factors that could serve as barriers to ART adherence using two instruments (BMQ-Q \& HIVASES). Based on the score on the instruments, a patient is identified for further intervention.

- Step 2: focused intervention on identified barriers is done at this stage with emphasis on interactive learning and feedback.

- Step 3: the final stage reflects the outcome of the intervention carried out at the early phase and indicates how successful the intervention was.

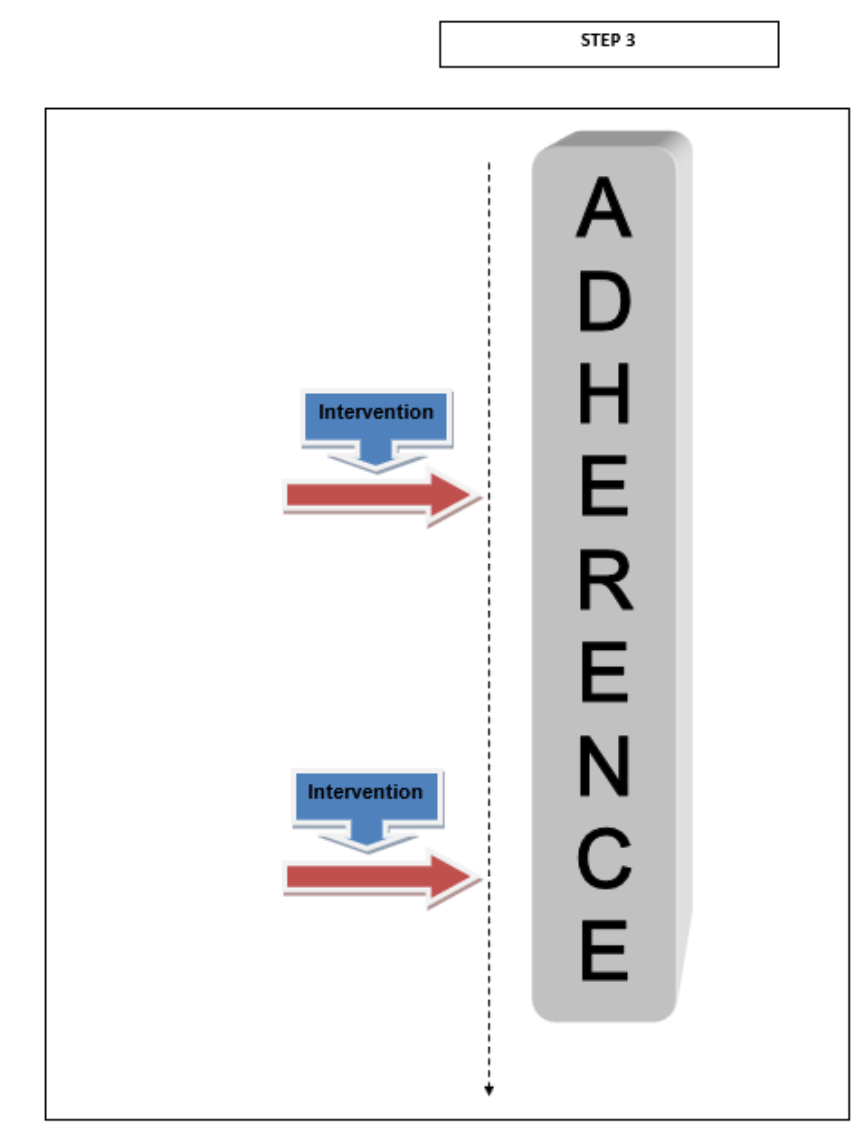

Fig. 5. Expected Outcome of focused interventions (enhanced ART adherence).

\section{CONCLUSION}

The theoretical framework for this research was based on two theories from the cognitive perspective of health behaviour, which are the Self-efficacy model (SEM) and the Health Belief Model (HBM).The cognitive perspective of health behaviour assisted the researcher to understand how HIV-infected patients on ART interpret and evaluate situations around them as well as their self-conception as to whether they will have self-efficacy in adhering to treatment. The broad overarching theoretical framework behind this research was aimed at explaining how HIV patients on ART conceptualize threats to their health (HIV/AIDS disease); how they conceptualize one specific solution to the health threat (treatment or medication use) and why actual medication use behaviour may or may not take place. This framework for enhancing ART adherence among HIV patients focuses on empowering the patients with the necessary skills. This will assist them to attain competence in self-efficacy and possessing stronger beliefs about medications that will result in better adherence to ART which will subsequently result in good clinical outcomes and reduced morbidity associated with HIV/AIDS. It is recommended that the framework be used in conjunction with other existing modalities of enhancing ART adherence for better results. 


\section{REFERENCES}

[1] Munro S, Lewin S, Swart T \& Volmink J. A review of health behaviour theories: how useful are these for developing interventions to promote long-term medication adherence for TB and HIV/AIDS. BMC Public Health 2007; 7:104.

[2] Rimer BK \& Glanz K. Theory at a Glance: Application to Health Promotion and Health Behaviour (2nd Edition). National Cancer Institute. Available online: http://www.cancer.gov/cancertopics/cancerlibrary/theory.pdf.

[3] Bandura A. Health promotion by social cognitive means. Health Education \& Behaviour. 2004; 31(2):143-164.

[4] Ogedegbe G, Mancuso CA, Allegrante JP \& Charlson ME. Development, and evaluation of a medication adherence self-efficacy scale in hypertensive African American patients. Journal of Clinical Epidemiology 2003; 56:520-529.

[5] Sleath, B, Blalock, SJ, Robin, A, Hartnett, ME, Covert, D, DeVellis, B \& Giangiacomo, A. Development of an instrument to measure glaucoma medication self-efficacy and outcome expectations. Eye 2010; 24:624-631.

[6] Zebracki K \& Drotar D. Outcome expectancy and self-efficacy in adolescent asthma self-management. Child Health Care 2004; 33(2):133-149.

[7] Hewlett, S. Cockshot, J. Kirwan, J, Barrett, J. Stamp, J. \& Haslock, I. Development and validation of a self-efficacy scale for use in British patients with rheumatoid arthritis (RASE). Rheumatology 2001; 40:1221-1230.

[8] Johnson, M. Neilands, T. Dilworth, S. Morin, S. Remien, R. \& Chesney, M. The role of self-efficacy in HIV treatment adherence: validation of the HIV treatment adherence self-efficacy scale (HIVASES). Journal of Behavioral Medicine 2007; 30(5): 359-370.

[9] Rudy, BJ. Murphy, DA. Harris, R. Muenz, PL. \& Ellen, J. (for the Adolescent Trials Network for HIV/AIDS Interventions). Patientrelated risks for non-adherence to antiretroviral therapy among HIVinfected youth in the United States: A study of prevalence and interactions. AIDS Patient Care \& STI 2009; 23(3):185-194.

[10] Redding, CA. Rossi, JS. Rossi, SR. Velicer, WF. Prochaska, JO. Health behaviour models. International Electronic Journal of Health Education 2000; 3: 180-193.

[11] Lennerling A \& Forsberg A. Self-reported non-adherence and beliefs about medication in a Swedish kidney transplant population. Open Journal of Nursing 2012; 6:41-46.

[12] Gonzalez, JS. Penedo, FJ. Llabre, MM. Duran, RE. Antoni, MH. Schneiderman, N. et al. Physical symptoms, beliefs about medications, negative mood, and long-term HIV medication adherence. Annal of Behavioral Medicine 2007; 34(1):46-55.

[13] Horne R, Weinman J \& Hankins M. The beliefs about medicines questionnaire: the development and evaluation of a new method for assessing the cognitive representation of medication. Psychology and Health 1999; 14:1-24.

[14] Magadza C, Radloff SE, Srinivas SC. The effect of an educational intervention on patients' knowledge about hypertension, beliefs about medicines, and adherence. Research in Social and Administrative Pharmacy 2009; 5(4):363-375.

[15] Adefolalu AO, Nkosi ZZ. Olorunju SS \& Masemola P. Self-efficacy, medication beliefs and adherence to antiretroviral therapy among patients attending a health facility in Pretoria. South African Family Practice 2014; 56(5):1-5.

[16] Adefolalu AO, Nkosi ZZ \& Olorunju SS. Patterns of adherence to scheduling and dietary instructions among patients on antiretroviral therapy in Pretoria, South Africa. Transactions of the Royal Society of Tropical Medicine \& Hygiene 2014; 108(9): 582-588.

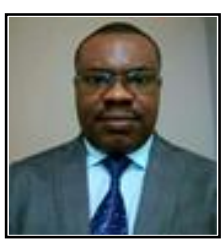

Dr. Adegoke O. Adefolalu, $M B C h B M P H P h D$ $F R S P H(U K)$ is with the School of Medicine at Sefako Makgatho Health Sciences University, South Africa, where he teaches Epidemiology and Research Methods within the Practice of Medicine integrated programme of the $\mathrm{MBCHB}$ curriculum. A Public health physician with interest in clinical education, some of his major research interest include clinical epidemiology, health behaviour and health determinants, international health, and curriculum integration in medical education. Dr Adefolalu is an established researcher; he is associate editor of two scientific publications and serves as reviewer for many medical journals. An astute health advocate, he mentors undergraduate and postgraduate students, and he is actively involved in faculty development. 